\title{
HAANTJES ALGEBRAS OF THE LAGRANGE TOP
}

\author{
GIORGIO TONDO
}

\begin{abstract}
A symplectic-Haantjes manifold and a Poisson-Haantjes manifold for the Lagrange top are studied and a set of Darboux-Haantjes coordinates are computed. Such coordinates are separation variables for the associated Hamilton-Jacobi equation.
\end{abstract}

\section{Contents}

1. Introduction

2. Nijenhuis and Haantjes torsion

3. Haantjes algebras

4. Poisson-Haantjes manifolds

5. The Lagrange top

5.1. Euler angles

5.2. Euler-Poisson coordinates

6. Future Perspectives

Acknowledgement

References

\section{INTRODUCTION}

In this article, we propose a new theoretical framework for dealing with integrable mechanical systems, and we illustrate it on the classical example of the Lagrange top. Precisely, we discuss such Hamiltonian system in the framework of the theory of Haantjes algebras, very recently introduced in 27. To this aim, the new geometric notion of Poisson-Haantjes $(P \mathcal{H})$ manifold is proposed, as a natural extension of the notion of symplectic-Haantjes $(\omega \mathcal{H})$ manifold, already introduced in [26]. The main idea is to replace the symplectic structure of a $\omega \mathcal{H}$ manifold with a Poisson (not invertible) bivector that fulfills a suitable algebraic compatibility condition with a Haantjes algebra of operators on the tangent bundle of the manifold. Besides, the dynamical notion of Magri-Haantjes chain [26] is generalized, to be adapted to the novel geometrical notion of $P \mathcal{H}$ manifold. These new structures can be used to describe bi-Hamiltonian chains of vector fields for Gelfand-Zakarevich (GZ) manifolds [10]. When such structures can be reduced to the symplectic leaves of the Poisson bivector, a $\omega \mathcal{H}$ structure is obtained and a set of Darboux-Haantjes coordinates can be computed. Such coordinates are separation variables for the Hamilton-Jacobi equation of the Hamiltonian systems belonging to a Magri-Haantjes chain. Here, we detail the Lagrange top, that is a GZ system of corank 2, whilst the discussion about the stationary flows of the KdV hierarchy (a GZ system of corank 1) will appear elsewhere. 
The notion of $P \mathcal{H}$ structures, is inspired, from one side, by the theory of PoissonNijenhuis structures [18, 13, from the other side by the notion of Haantjes manifolds [14, 15, 16, 17]. In our opinion, the new theory provides us a very flexible and unifying theoretical framework for dealing with the integrability and separability properties of Hamiltonian systems, and represents a formulation that completes the one offered by the Poisson-Nijenhuis geometry.

The paper is organized as follows. After a review, in Section 2, of the main algebraic structures needed in this work, we recall in Section 3 the concept of Haantjes algebras. In Section 4, we present the new notion of Poisson-Haantjes manifolds and of the related Magri-Haantjes chains. In Section 5, we apply the theory to the real and complex Lagrange top.

\section{NiJEnHUIS AND HAANTJES TORSION}

The natural frames $\left(\frac{\partial}{\partial x_{1}}, \ldots, \frac{\partial}{\partial x_{n}}\right)$ of local charts $\left(x_{1}, \ldots, x_{n}\right)$ in a differentiable manifold, being obviously integrable, can be characterized in a tensorial manner as eigen-distributions of a suitable class of $(1,1)$ tensor fields, i.e. the ones with vanishing Haantjes tensor. In this section, we review some basic results concerning the theory of such tensors. For a more complete treatment, see the original papers [11, 24, the related ones [25, 9], and the nice recent review [12].

Let $M$ be a differentiable manifold and $\boldsymbol{L}: T M \rightarrow T M$ be a $(1,1)$ tensor field, i.e., a field of linear operators on the tangent space at each point of $M$.

Definition 1. The Nijenhuis torsion of $\boldsymbol{L}$ is the skew-symmetric $(1,2)$ tensor field defined by

$$
\mathcal{T}_{\boldsymbol{L}}(X, Y):=\boldsymbol{L}^{2}[X, Y]+[\boldsymbol{L} X, \boldsymbol{L} Y]-\boldsymbol{L}([X, \boldsymbol{L} Y]+[\boldsymbol{L} X, Y]),
$$

where $X, Y \in T M$ and [, ] denotes the commutator of two vector fields.

In local coordinates $\boldsymbol{x}=\left(x_{1}, \ldots, x_{n}\right)$, the Nijenhuis torsion can be written in the form

$$
\left(\mathcal{T}_{\boldsymbol{L}}\right)_{j k}^{i}=\sum_{\alpha=1}^{n}\left(\frac{\partial \boldsymbol{L}_{k}^{i}}{\partial x^{\alpha}} \boldsymbol{L}_{j}^{\alpha}-\frac{\partial \boldsymbol{L}_{j}^{i}}{\partial x^{\alpha}} \boldsymbol{L}_{k}^{\alpha}+\left(\frac{\partial \boldsymbol{L}_{j}^{\alpha}}{\partial x^{k}}-\frac{\partial \boldsymbol{L}_{k}^{\alpha}}{\partial x^{j}}\right) \boldsymbol{L}_{\alpha}^{i}\right)
$$

amounting to $n^{2}(n-1) / 2$ independent components.

Definition 2. The Haantjes torsion associated with $\boldsymbol{L}$ is the $(1,2)$ tensor field defined by

$$
\mathcal{H}_{\boldsymbol{L}}(X, Y):=\boldsymbol{L}^{2} \mathcal{T}_{\boldsymbol{L}}(X, Y)+\mathcal{T}_{\boldsymbol{L}}(\boldsymbol{L} X, \boldsymbol{L} Y)-\boldsymbol{L}\left(\mathcal{T}_{\boldsymbol{L}}(X, \boldsymbol{L} Y)+\mathcal{T}_{\boldsymbol{L}}(\boldsymbol{L} X, Y)\right)
$$

The skew-symmetry of the Nijenhuis torsion implies that the Haantjes tensor is also skew-symmetric. Its local expression is

$$
\left(\mathcal{H}_{\boldsymbol{L}}\right)_{j k}^{i}=\sum_{\alpha, \beta=1}^{n}\left(\boldsymbol{L}_{\alpha}^{i} \boldsymbol{L}_{\beta}^{\alpha}\left(\mathcal{T}_{\boldsymbol{L}}\right)_{j k}^{\beta}+\left(\mathcal{T}_{\boldsymbol{L}}\right)_{\alpha \beta}^{i} \boldsymbol{L}_{j}^{\alpha} \boldsymbol{L}_{k}^{\beta}-\boldsymbol{L}_{\alpha}^{i}\left(\left(\mathcal{T}_{\boldsymbol{L}}\right)_{\beta k}^{\alpha} \boldsymbol{L}_{j}^{\beta}+\left(\mathcal{T}_{\boldsymbol{L}}\right)_{j \beta}^{\alpha} \boldsymbol{L}_{k}^{\beta}\right)\right)
$$

We shall consider a case, in which the computation of the Haantjes torsion will be particularly simple [26]. 
Proposition 3. Let $\boldsymbol{L}$ be a smooth field of operators. If there exists a local chart $\left\{U,\left(x_{1}, \ldots, x_{n}\right)\right\}$ where $\boldsymbol{L}$ takes the diagonal form

$$
\boldsymbol{L}(\boldsymbol{x})=\sum_{i=1}^{n} l_{i}(\boldsymbol{x}) \frac{\partial}{\partial x_{i}} \otimes \mathrm{d} x_{i},
$$

then the Haantjes tensor of $\boldsymbol{L}$ vanishes.

Due to the relevance of the Haantjes (Nijenhuis) vanishing condition, we propose the following definition.

Definition 4. A Haantjes (Nijenhuis) operator is a field of operators whose Haantjes (Nijenhuis) tensor identically vanishes.

It has been proved in the following proposition that a single Haantjes operator generates an algebra of Haantjes operators over the ring of smooth functions on $M$. This is not the case for a Nijenhuis operator $\boldsymbol{N}$ since a polynomial in $\boldsymbol{N}$ with coefficients $a_{j} \in C^{\infty}(M)$, is not necessarily a Nijenhuis operator.

Proposition 5. [5]. Let $\boldsymbol{L}$ be a Haantjes operator in $M$. Then for any polynomial in $\boldsymbol{L}$ with coefficients $a_{j} \in C^{\infty}(M)$, the associated Haantjes tensor vanishes, i.e.

$$
\mathcal{H}_{\boldsymbol{L}}(X, Y)=0 \Longrightarrow \mathcal{H}_{\left(\sum_{j} a_{j}(\boldsymbol{x}) \boldsymbol{L}^{j}\right)}(X, Y)=0 .
$$

Proof. See Corollary 3.3, p. 1136 of $[5$.

\section{HaAntJes Algebras}

In this section we recall the notion of Haantjes algebra and the class of cyclic Haantjes algebras, very recently introduced in [27.

Definition 6. A Haantjes algebra of rank $m$ is a pair $(M, \mathscr{H})$ which satisfies the following conditions:

- $M$ is a differentiable manifold of dimension $n$;

- $\mathscr{H}$ is a set of Haantjes operators $\boldsymbol{K}_{i}: T M \rightarrow T M$ that generates

- a free module of rank $m$ over the ring of smooth functions on $M$

(8)

$$
\mathcal{H}_{\left(f \boldsymbol{K}_{i}+g \boldsymbol{K}_{j}\right)}(X, Y)=0, \quad \forall X, Y \in T M, \forall f, g \in C^{\infty}(M) ;
$$

- a ring w.r.t. the composition operation

$$
\mathcal{H}_{\left(\boldsymbol{K}_{i} \boldsymbol{K}_{j}\right)}(X, Y)=\mathcal{H}_{\left(\boldsymbol{K}_{j} \boldsymbol{K}_{i}\right)}(X, Y)=0, \quad \forall \boldsymbol{K}_{i}, \boldsymbol{K}_{j} \in \mathscr{H}, \quad \forall X, Y \in T M
$$

The assumption (7), (8), ensure that the set $\mathscr{H}$ is an associative algebra of Haantjes operators. In addition, if

$$
\boldsymbol{K}_{i} \boldsymbol{K}_{j}=\boldsymbol{K}_{i} \boldsymbol{K}_{j} \quad \forall \boldsymbol{K}_{i}, \boldsymbol{K}_{j} \in \mathscr{H},
$$

the algebra $\mathscr{H}$ will be said an Abelian Haantjes algebra.

Remark 7. The conditions of Definition [6] are apparently very demanding and difficult to solve. However, a class of natural solutions is given, in a local chart $\left\{U, \boldsymbol{x}=\left(x_{1}, \ldots, x_{n}\right)\right\}$, by each operator of the form

$$
\boldsymbol{K}=\sum_{i=1}^{n} l_{i}(\boldsymbol{x}) \frac{\partial}{\partial x_{i}} \otimes \mathrm{d} x_{i}
$$


The diagonal operators $\boldsymbol{K}$ have their Haantjes tensor vanishing and satisfy the differential compatibility condition (77) by virtue of Proposition [3. Moreover, they form a commutative ring, therefore they fulfill Eqs. (8). In fact, such operators generate an algebraic structure that we shall call a diagonal Haantjes algebra.

A particular but especially relevant class of Haantjes algebras is given by the ones generated by a single Haantjes operator $\boldsymbol{L}: T M \mapsto T M$. In fact, one can construct directly a Haantjes algebra $\mathcal{L}$, of $\operatorname{rank} \leq n=\operatorname{dim}(M)$, by choosing as a set of generators the first $(n-1)$ powers of $\boldsymbol{L}$ together with $\boldsymbol{L}^{0}:=\boldsymbol{I}$

$$
\mathcal{L}(\boldsymbol{L}):=\operatorname{Span}\left\{\boldsymbol{I}, \boldsymbol{L}, \boldsymbol{L}^{2}, \boldsymbol{L}^{n-1}\right\} .
$$

We shall call these algebras cyclic Haantjes algebras. Their rank is equal to the degree of the minimal polynomial of $\boldsymbol{L}$.

A natural question is to establish when a given Haantjes algebra can be generated by a single Haantjes operator, giving rise to a cyclic Haantjes algebra. This problem has been investigated in 27. starting from the following

Definition 8. Let $(M, \mathscr{H})$ be a Haantjes algebra of rank $m$. An operator $\boldsymbol{L}$ will be called a cyclic generator of $\mathscr{H}$ if

$$
\mathscr{H} \equiv \mathcal{L}(\boldsymbol{L})
$$

The basis

$$
\mathcal{B}_{\text {cyc }}=\left\{\boldsymbol{I}, \boldsymbol{L}, \boldsymbol{L}^{2}, \boldsymbol{L}^{m-1}\right\}
$$

will be called a cyclic basis of $\mathscr{H}$ and allows us to represent each Haantjes operator $\boldsymbol{K} \in \mathscr{H}$ as a polynomial field in $\boldsymbol{L}$ of degree at most (m-1), i.e.

$$
\boldsymbol{K}=p_{\boldsymbol{K}}(\boldsymbol{x}, \boldsymbol{L})=\sum_{i=0}^{m-1} a_{i}(\boldsymbol{x}) \boldsymbol{L}^{i}
$$

where $a_{i}(\boldsymbol{x})$ are smooth functions in $M$.

\section{Poisson-HaAntJes MANifolds}

In order to deal with GZ systems, we need to extend the notion of symplecticHaantjes manifold $(\omega \mathcal{H})$ already introduced in [26. Here we propose the new notion of Poisson-Haantjes $(P \mathcal{H})$ manifold.

As usual, the transposed operator $\boldsymbol{K}^{T}: T^{*} M \rightarrow T^{*} M$ is defined as the transposed linear map of $\boldsymbol{K}: T M \rightarrow T M$, with respect to the natural pairing between a vector space and its dual space.

Definition 9. A Poisson-Haantjes manifold is a triple $(M, P, \mathscr{H})$ that satisfies the following conditions

i) $M$ is a differentiable manifold;

ii) $P: T M^{*} \rightarrow T M$ is a Poisson bivector in $M$;

iii) $\mathscr{H}$ is an Abelian Haantjes algebra;

iv) $(P, \mathscr{H})$ are algebraically compatible in the sense that $\boldsymbol{K} P=P \boldsymbol{K}^{T}, \forall \boldsymbol{K} \in$ $\mathscr{H}$.

As a consequence of the above conditions, we get the following simple proposition that turns out to be crucial for many results of the present theory. 
Proposition 10. In a given a $P \mathcal{H}$ manifold, any composed operator, $\boldsymbol{K}_{i} P, \boldsymbol{K}_{i} P \boldsymbol{K}_{j}^{T}$, $\left(\boldsymbol{K}_{\alpha}-f(\boldsymbol{x}) \boldsymbol{I}\right)^{r} P, r \in \mathbb{N}$, is skew symmetric.

Remark 11. The class of $\omega \mathcal{H}$ manifolds coincides with the class of PH manifolds of even dimension, with an invertible Poisson bivector. In fact, as in this case $\Omega=P^{-1}$ is a symplectic operator, the compatibility condition $\boldsymbol{K} P=P \boldsymbol{K}^{T}$ is equivalent to the compatibility condition $\Omega \boldsymbol{K}=\boldsymbol{K}^{T} \Omega$, required in $\omega \mathcal{H}$ manifolds.

We show a paradigmatic example of $P \mathcal{H}$ manifold with a cyclic Haantjes algebra, that later will be used to describe a Haantjes algebra of the Lagrange top.

Example 12. Let $(M, P, \boldsymbol{N})$ be a Poisson-Nijenhuis (PN) manifold, that is, a manifold endowed with a Poisson bivector $P$ and a Nijenhuis operator $\boldsymbol{N}$ that satisfy the following compatibility conditions

$$
\begin{aligned}
\boldsymbol{N} P-P \boldsymbol{N}^{T} & =0 \\
R(P, \boldsymbol{N})(\alpha, Y) & =0
\end{aligned} \quad \forall \alpha \in T^{*} M, \forall Y \in T M,
$$

where $R(P, \boldsymbol{N})$ is the $(2+1)$ tensor field defined in [18] by

$$
R(P, \boldsymbol{N})(\alpha, Y)=\mathcal{L}_{P \alpha}(\boldsymbol{N}) Y-P\left(\mathcal{L}_{Y}\left(\boldsymbol{N}^{T} \alpha\right)-\mathcal{L}_{N Y} \alpha\right),
$$

$\left(\mathcal{L}_{Y}\right.$ denotes the Lie derivative with respect the vector field $\left.Y\right)$. Let us suppose that the Nijenhuis operator $\boldsymbol{N}$ has its minimal polynomial of degree $\mathrm{m}$. Then, the PN manifold $M$ has a standard $P \mathcal{H}$ structure, given by

$$
\left(M, P, \boldsymbol{K}_{1}=\boldsymbol{I}, \boldsymbol{K}_{2}=\boldsymbol{N}, \ldots,, \boldsymbol{K}_{m}=\boldsymbol{N}^{\boldsymbol{m}-\mathbf{1}}\right),
$$

with a Haantjes algebra of rank $\mathrm{m} \leq \operatorname{dim}(M)$. In fact, each Nijenhuis operator $\boldsymbol{N}$ is also a Haantjes operator, therefore generates the cyclic Haantjes algebra $\mathcal{L}(\boldsymbol{N})$. Moreover, the algebraic compatibility condition (13) assures that for all Haantjes operators

$$
\boldsymbol{K}=p_{\boldsymbol{K}}(\boldsymbol{x}, \boldsymbol{N})=\sum_{i=0}^{m-1} a_{i}(\boldsymbol{x}) \boldsymbol{N}^{i}
$$

the condition iv) of Def. 9 is fulfilled.

In addition, the differential condition (14) implies that

$$
R(P, \boldsymbol{K})(\alpha, Y)-\left(\sum_{i=0}^{m-1}\left(\left(X_{i} \wedge N^{i} Y\right)-Y\left(a_{i}\right) \boldsymbol{N}^{i} P\right)\right) \alpha=0
$$

$\forall j \in \mathbb{N}, \forall \alpha \in T^{*} M, \forall Y \in T M$, where $X_{i}:=P \mathrm{~d} a_{i}$ are the Hamiltonian vector fields with Hamiltonian functions $a_{i}$.

We also generalize the concept of Magri-Haantjes chain, introduced in [26] under the name of Lenard-Haantjes chains.

Definition 13. Let $(M, P, \mathscr{H})$ be a Poisson-Haantjes manifold. A function $H \in$ $C^{\infty}(M)$ is said to generate a Magri-Haantjes chain of 1-forms if

$$
\mathrm{d}\left(\boldsymbol{K}_{i}^{T} \mathrm{~d} H\right)=0, \quad i=1, \ldots, m,
$$

for some basis $\left\{\boldsymbol{K}_{1}, \ldots, \boldsymbol{K}_{m}\right\}$ of $\mathscr{H}$. The (locally) exact 1-forms $\mathrm{d} H_{i}$ such that

$$
\mathrm{d} H_{i}=\boldsymbol{K}_{i}^{T} \mathrm{~d} H \quad i=1, \ldots, m,
$$

are called the elements of the Magri-Haantjes chain, of length $m$, generated by $H$.

The relevance of Magri-Haantjes chains is due to the following 
Lemma 14. Let $(M, P, \mathscr{H})$ be a Poisson-Haantjes manifold. The functions $H_{i}$ whose differentials belong to all Magri-Haantjes chains generated by a single function $H$ are in involution w.r.t. the Poisson bracket defined by $P$. In fact,

$$
\left\{H_{i}, H_{j}\right\}=<d H_{i}, P d H_{j}>=<\boldsymbol{K}_{i}^{T} d H, P \boldsymbol{K}_{j}^{T} d H>=<d H, \boldsymbol{K}_{i} P \boldsymbol{K}_{j}^{T} d H>\stackrel{\text { Prop. }}{\underline{10}} 0
$$

Definition 15. Let $(M, P, \mathscr{H})$ be a Poisson-Haantjes manifold. A vector field $Y$ is said to generate a Magri-Haantjes chain of vector fields if the vector fields defined by

$$
Y_{i}:=\boldsymbol{K}_{i} Y, \quad i=1, \ldots, m
$$

for some basis $\left\{\boldsymbol{K}_{1}, \ldots, \boldsymbol{K}_{m}\right\}$, commute among each others.

Remark 16. Let us note that, thanks to the compatibility condition iv) in Definition 9, to every Magri-Haantjes chain of 1-forms generated by a function $H$ corresponds a Magri-Haantjes chain of Hamiltonian vector fields $X_{H_{i}}=P \mathrm{~d} H_{i}$ generated by $X_{H}=P \mathrm{~d} H$. Moreover, the Hamiltonian vector fields belonging to different chains generated by the same Hamiltonian vector field $X_{H}$ commute among each others.

In [26, it has been shown that, given a $\omega \mathcal{H}$ manifold and a function $H$, the existence of a Magri-Haantjes chain generated by $H$ is equivalent to the Frobenius integrability of the co-distribution

$$
\boldsymbol{K}_{i}^{T} \mathrm{~d} H \quad i=1, \ldots m .
$$

In this paper, we limit ourselves to exhibit the example of the Magri-Haantjes chain of the Lagrange top, leaving the finding of the conditions assuring the existence of Magri-Haantjes chains in $P \mathcal{H}$ manifolds to future work.

\section{The LAGRANGE TOP}

The classical Lagrange top is a heavy symmetric top, that is, a symmetric rigid body with a fixed point $O$, immersed in the uniform gravity field $\vec{\gamma}$. It admits different geometric formulations in the framework of the bi-Hamiltonian theory [22, 23, 30].

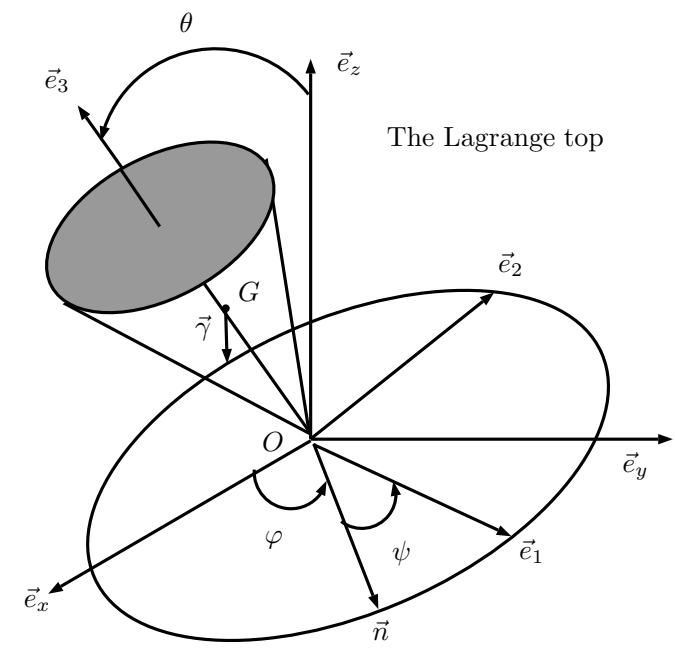


5.1. Euler angles. In the phase space $M=T^{*}(S O(3))$ one can choose as local coordinates the classical Euler angles and conjugate momenta $\left(\varphi, \theta, \psi, p_{\varphi}, p_{\theta}, p_{\psi}\right)$. In such a chart, the Hamiltonian function of the Lagrange top takes the form

$$
H=\frac{1}{2 \mathcal{A}}\left(p_{\theta}^{2}+\frac{\left(p_{\varphi}-p_{\psi} \cos \theta\right)^{2}}{\sin ^{2} \theta}+\frac{1}{c} p_{\psi}^{2}\right)+\mu g a \cos \theta
$$

and the Hamilton equations of the motion are

$$
\begin{aligned}
\dot{\varphi} & =\frac{1}{\mathcal{A}} \frac{p_{\varphi}-p_{\psi} \cos \theta}{\sin ^{2} \theta} \\
\dot{\theta} & =\frac{1}{\mathcal{A}} p_{\theta} \\
\dot{\psi} & =\frac{1}{c \mathcal{A}} p_{\theta} \\
\dot{p}_{\varphi} & =0 \\
\dot{p}_{\theta} & =-\frac{\partial}{\partial \theta} \frac{\left(p_{\varphi}-p_{\psi} \cos \theta\right)^{2}}{\sin ^{2} \theta}+\mu g a \sin \theta \\
\dot{p}_{\psi} & =0
\end{aligned}
$$

where $\mathcal{A}$ and $c \mathcal{A}$ are, respectively, the inertia momenta w.r.t. every axis in the equatorial plane and the symmetry axis, $\mu$ is the mass of the top, $g$ the gravity acceleration, $a$ the coordinate of the mass center $G$ along the symmetry axis. It is evident from the Hamiltonian function and the equations of the motion that the Lagrange top admits the three integrals of motion

$$
H_{1}=H, \quad H_{2}=p_{\varphi}, \quad H_{3}=p_{\psi},
$$

that are, the energy and the components of the angular momentum along the vertical and the symmetry axis, respectively. Moreover, it is well known that the coordinates $\left(\varphi, \theta, \psi, p_{\varphi}, p_{\theta}, p_{\psi}\right)$ are separation variables for the Hamilton-Jacobi equation associated to $H$. From our point of view, it is worth of interest to show that even this very classical system, as well as every Hamiltonian separable system, can be described in the framework of $\omega \mathcal{H}$ Haantjes manifolds, according to Theorem 57 of [26]. A Haantjes algebra for the Lagrange top can be easily computed, whose basis is

$$
\begin{aligned}
& \boldsymbol{K}_{1}=\boldsymbol{I} \\
& \boldsymbol{K}_{2}=\frac{\mathcal{A} \sin ^{2} \theta}{p_{\varphi}-p_{\psi} \cos \theta}\left(\frac{\partial}{\partial \varphi} \otimes \mathrm{d} \varphi+\frac{\partial}{\partial p_{\varphi}} \otimes \mathrm{d} p_{\varphi}\right) \\
& \boldsymbol{K}_{3}=-\frac{\mathcal{A} \sin ^{2} \theta}{\cos \theta\left(p_{\varphi}-p_{\psi} \cos \theta\right)}\left(\frac{\partial}{\partial \theta} \otimes \mathrm{d} \theta+\frac{\partial}{\partial p_{\theta}} \otimes \mathrm{d} p_{\theta}\right) .
\end{aligned}
$$

In fact, the action of the (transpose of the) Haantjes operators $\boldsymbol{K}_{1}, \boldsymbol{K}_{2}, \boldsymbol{K}_{3}$ on the gradient of the Hamiltonian function (19) produces the Magri-Haantjes chain of the (gradients of the) three integrals of motion

$$
\boldsymbol{K}_{1}^{T} \mathrm{~d} H=\mathrm{d} H_{1}, \quad \boldsymbol{K}_{2}^{T} \mathrm{~d} H=\mathrm{d} H_{2}, \quad \boldsymbol{K}_{3}^{T} \mathrm{~d} H=\mathrm{d} H_{3} .
$$

The fact that the Haantjes operators take a diagonal form in the Euler chart $\left(\varphi, \theta, \psi, p_{\varphi}, p_{\theta}, p_{\psi}\right)$, means that the Haantjes algebra is diagonal and the Euler coordinates are Darboux-Haantjes coordinates for it. 
More interesting and hard to solve is the problem to construct a Haantjes algebra directly in the physical coordinates, and, afterwards determining a set of DarbouxHaantjes coordinates that are separation variables according to Theorem 59 of [26]. In the next Section, we will show how to proceed in this example, starting with the tri-Hamiltonian formulation of the Lagrange top and reducing it to the symplectic leaves of one of its Poisson bivectors.

5.2. Euler-Poisson coordinates. An alternative formulation of the Lagrange top is based on the Euler-Poisson equation that are, roughly speaking, the equation of the motion projected onto the comoving reference frame $\left(\vec{e}_{1}, \vec{e}_{2}, \vec{e}_{3}\right)$. Let us consider the phase space $M:=\{m \mid m=(\vec{\omega}, \vec{\gamma})\}$, where $\vec{\omega}=\omega_{1} \vec{e}_{1}+\omega_{2} \vec{e}_{2}+\omega_{3} \vec{e}_{3}$ is the angular velocity of the top, $\vec{\gamma}=\gamma_{1} \vec{e}_{1}+\vec{e}_{2} \gamma_{2}+\gamma_{3} \vec{e}_{3}$ the vertical unit vector (the Poisson vector), $G-O=a \vec{e}_{3}$ the vector of the center of mass and $J=\operatorname{diag}(\mathcal{A}, \mathcal{A}, c \mathcal{A})$ the principal inertia matrix. In such a formulation, the equations of the motion $\dot{m}=X_{L}(m)$ are the Euler equations coupled with the Poisson equations, so that the Lagrange vector field is given by

$$
X_{L}(m)=\left[\begin{array}{c}
(1-c) \omega_{2} \omega_{3}-\gamma_{2} \\
-(1-c) \omega_{3} \omega_{1}+\gamma_{1} \\
0 \\
\gamma_{2} \omega_{3}-\gamma_{3} \omega_{2} \\
\gamma_{3} \omega_{1}-\gamma_{1} \omega_{3} \\
\gamma_{1} \omega_{2}-\gamma_{2} \omega_{1}
\end{array}\right]
$$

where the normalization $\mu g a / \mathcal{A}=1$ has been chosen.

5.2.1. The tri-Hamiltonian formulation of the Lagrange top. The vector field (21) admits a tri-Hamiltonian formulation (see 22] and reference therein) w.r.t. the three non invertible Poisson bivectors

$$
P_{0}=\left[\begin{array}{ll}
0 & B \\
B & C
\end{array}\right], P_{1}=\left[\begin{array}{cc}
-B & 0 \\
0 & \Gamma
\end{array}\right], P_{2}=\left[\begin{array}{cc}
T & R \\
-R^{T} & 0
\end{array}\right],
$$

where

$$
\begin{gathered}
B=\left[\begin{array}{ccc}
0 & -1 & 0 \\
1 & 0 & 0 \\
0 & 0 & 0
\end{array}\right], C=\left[\begin{array}{ccc}
0 & c \omega_{3} & -\omega_{2} \\
-c \omega_{3} & 0 & \omega_{1} \\
\omega_{2} & -\omega_{1} & 0
\end{array}\right] \\
\Gamma=\left[\begin{array}{ccc}
0 & \gamma_{3} & -\gamma_{2} \\
-\gamma_{3} & 0 & \gamma_{1} \\
\gamma_{2} & -\gamma_{1} & 0
\end{array}\right], R=\left[\begin{array}{cccc}
0 & -\gamma_{3} & \gamma_{2} \\
\gamma_{3} & 0 & -\gamma_{1} \\
-\gamma_{2} / c & \gamma_{1} / c & 0
\end{array}\right] \\
T=\left[\begin{array}{ccc}
0 & -c \omega_{3} & \omega_{2} / c \\
c \omega_{3} & 0 & -\omega_{1} / c \\
-\omega_{2} / c & \omega_{1} / c & 0
\end{array}\right],
\end{gathered}
$$

and the Hamiltonian functions $\left(h_{0}, h_{1}, h_{2}\right)$

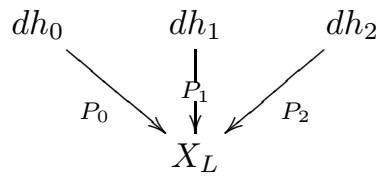

defined by

$$
h_{0}=\frac{1}{2} F_{4}+(c-1) F_{1} F_{3}, \quad h_{1}=-F_{3}-(c-1) F_{1} F_{2}, \quad h_{2}=F_{2} .
$$


The functions $F_{1}, F_{2}, F_{3}, F_{4}$ are the integrals of motion given by

$$
\begin{gathered}
F_{1}=\omega_{3}, \quad F_{2}=\frac{1}{2}\left(\omega_{1}^{2}+\omega_{2}^{2}+c \omega_{3}^{2}\right)-\gamma_{3}, \\
F_{3}=\omega_{1} \gamma_{1}+\omega_{2} \gamma_{2}+c \omega_{3} \gamma_{3}, \quad F_{4}=\gamma_{1}^{2}+\gamma_{2}^{2}+\gamma_{3}^{2} .
\end{gathered}
$$

The three Poisson bivectors $\left(P_{0}, P_{1}, P_{2}\right)$ generate three Poisson pencils

$$
P_{0}-\lambda P_{1}, \quad P_{1}-\lambda P_{2}, \quad P_{0}-\lambda P_{2},
$$

that possess two polynomial Casimir functions each [23]. Below, we concentrate on the Casimir function of the Poisson pencil $P_{0}-\lambda P_{1}$.

5.2.2. A Gelfand-Zakharevich system of co-rank 2. The Poisson pencil $P_{0}-\lambda P_{1}$ possess two polynomial Casimir functions $H(\lambda)^{(1)}=H_{0}^{(1)}=F_{1}$ and $H(\lambda)^{(2)}=$ $H_{0}^{(2)} \lambda^{2}+H_{1}^{(2)} \lambda+H_{2}=\frac{F_{4}}{2} \lambda^{2}-F_{3} \lambda+F_{2}$, of length 1 and 3 respectively. They can be represented graphically in the following way
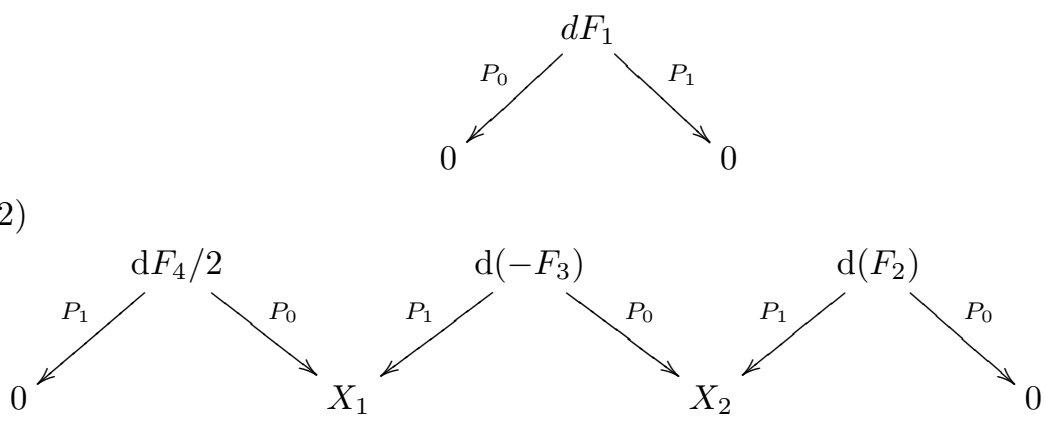

The vector fields $X_{1}, X_{2}$ are bi-Hamiltonian vector fields as

$$
X_{i}=P_{0} \mathrm{~d} H_{i-1}^{(2)}=P_{1} \mathrm{~d} H_{i}^{(2)}, \quad i=1,2 .
$$

Moreover, the vector field (21) of the Lagrange top can be formulated as

$$
X_{L}=X_{1}-(c-1) F_{1} X_{2},
$$

therefore defining a system of Gelfand-Zakharevich type of co-rank 2.

5.2.3. The reduction of the Poisson pencil. Without loss of generality, we fix a Poisson bivector inside the Poisson pencil, say $P_{1}$, and, in order to getting rid of its Casimir functions, we perform a reduction to its symplectic leaves

$$
S_{1}:=\left\{F_{1}=C_{1}, F_{4}=C_{4}\right\} .
$$

To this aim, it is convenient to introduce complex coordinates in $M$ adapted to such a reduction 22

$$
x_{1}=-c \omega_{3}+i \omega_{2}, \quad x_{2}=\gamma_{3}-i \gamma_{2}, \quad y_{1}=\omega_{1}, \quad y_{2}=-\gamma_{1}, \quad F_{1}, \quad F_{4},
$$

in which the Poisson bivectors take the following simple form

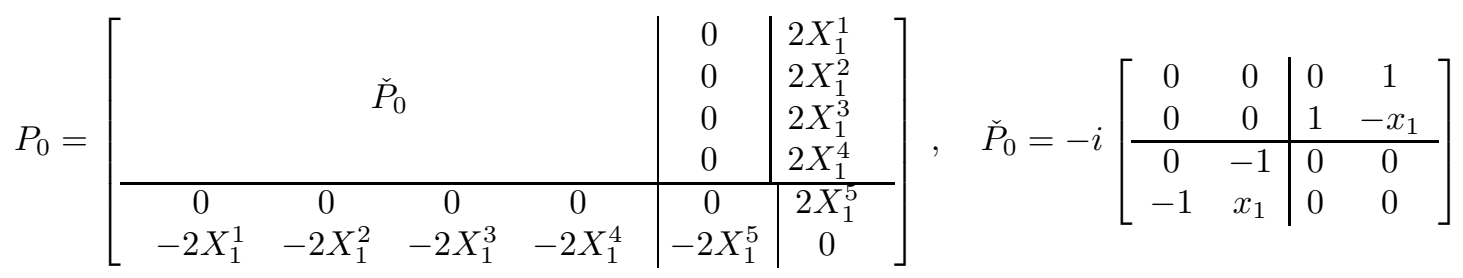




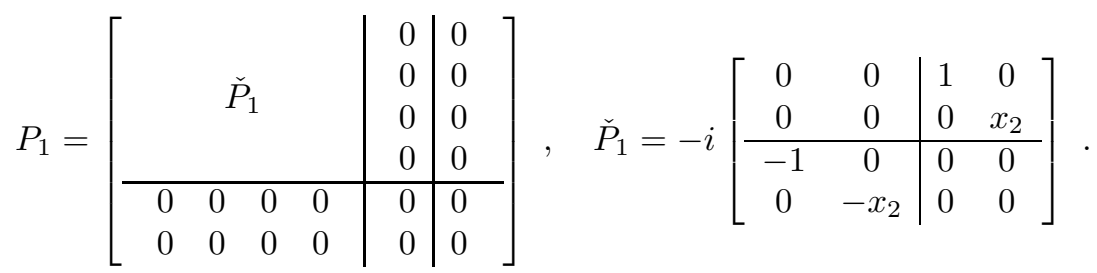

It is evident, that the Poisson bivector $P_{0}$ cannot be restricted to $S_{1}$, unlike $P_{1}$. So, we perform a reduction procedure introduced in [7. It is a highly non trivial generalization of the Marsden-Ratiu method [19] and consists in an ingenious deformation of the Poisson bivector $P_{0}$, by means of a suitable family of vector fields transversal to the symplectic leaves of $P_{1}$. Such a deformation assures that the deformed Poisson pencil shares the same axis with the old one and can be restricted to $S_{1}$.

5.2.4. Deformation. We choose the two vector fields

$$
Z_{1}=\frac{\partial}{\partial F_{1}}, \quad Z_{2}=2 \frac{\partial}{\partial F_{4}}
$$

normalized as

$$
Z_{i}\left(H_{0}^{(j)}\right)=\delta_{i}^{j}, \quad i, j=1,2 .
$$

As they fulfill the equations

$$
\mathcal{L}_{Z_{i}}\left(P_{1}\right)=0 \quad \mathcal{L}_{Z_{i}}\left(P_{0}\right)=\left[Z_{i}, X_{1}\right] \wedge Z_{2} \quad i=1,2,
$$

they can deform the Poisson bivector $P_{0}$ into the new Poisson bivector

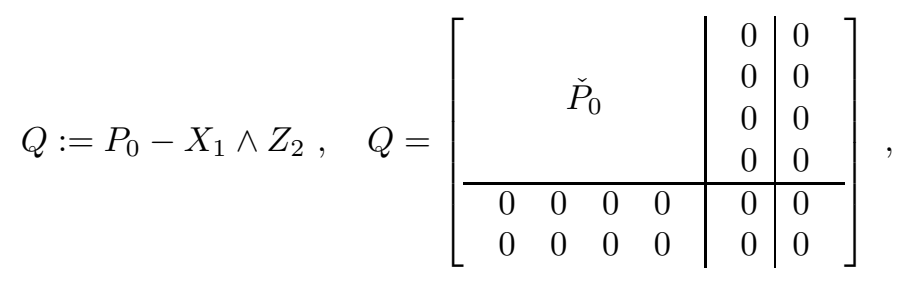

that can be restricted to $S_{1}$ and its restriction is $\check{Q}=\check{P}_{0}$.

5.2.5. Haantjes algebra. There are different manners of endowing the manifold $M$ with a Haantjes algebra compatible with $P_{1}$. In this section, we limit ourselves to show one of them, leaving a more general discussion to future work. In order to construct a Haantjes algebra for the bi-Hamiltonian chain (22), which will be preserved after the restriction to $S_{1}$, we look for a Nijenhuis operator $\boldsymbol{N}$ that plays the role of a cyclic generator for a Haantjes algebra of low rank, that is $2 \leq m \leq 3$. Precisely, we require that $\boldsymbol{N}$ :

i) factorizes the deformed Poisson bivector

$$
Q=N P_{1}
$$

ii) has its restriction $\check{N}$ to $S_{1}$ equal to

$$
\check{N}=\check{Q}_{1}^{-1}=\check{P}_{0} \check{P}_{1}^{-1} ;
$$


iii) is a cyclic generator of a Haantjes algebra that provides the following MagriHaantjes chain of vector fields generated by $X_{1}$

$$
\boldsymbol{K}_{i} X_{1}=X_{i}, \quad i=1,2,3,
$$

where $X_{3}=0$.

In other words, denoted with $\Phi$ the immersion of $S_{1}$ in $M$, and with $\Phi_{*}$ and $\Phi^{*}$ its pullback and pushforward respectively, we search for an operator $\boldsymbol{N}: T M \rightarrow T M$ that solves the system

$$
\begin{aligned}
\boldsymbol{N} P_{1} & =P_{0}-X_{1} \wedge Z_{2} \\
\Phi_{*} \boldsymbol{N} \Phi^{*} & =\check{P}_{0} \check{P}_{1}^{-1} \\
\left(d_{1} \boldsymbol{I}+e_{1} \boldsymbol{N}+f_{1} \boldsymbol{N}^{2}\right) X_{1} & =X_{1} \\
\left(d_{2} \boldsymbol{I}+e_{2} \boldsymbol{N}+f_{2} \boldsymbol{N}^{2}\right) X_{1} & =X_{2} \\
\left(d_{3} \boldsymbol{I}+e_{3} \boldsymbol{N}+f_{3} \boldsymbol{N}^{2}\right) X_{1} & =X_{3} \\
\tau(\boldsymbol{N}) & =0,
\end{aligned}
$$

together with the unknown functions $\left(d_{i}, e_{i}, f_{i}\right), i=1,2,3$. Such a system can be decoupled in order to firstly determine the unknown functions $\left(d_{i}, e_{i}, f_{i}\right)$. In fact, applying both terms of Eq. (30) to the gradients of the bi-Hamiltonian chain (22), and taking into account the fact that the Hamiltonian functions $H_{j}$ are integrals of motion for $X_{1}$, one finds that the bi-Hamiltonian vector fields (23) must fulfill the system

$$
\boldsymbol{N} X_{j}=X_{j+1}+X_{1}\left(H_{j}\right) Z_{2}-Z_{2}\left(H_{j}^{(2)}\right) X_{1}, \quad X_{0}:=0, \quad j=0,1,2 .
$$

By solving recursively such a system w.r.t. $X_{j+1}$, only in terms of the vector field $X_{1}$, a unique solution is found for the unknown functions $\left(d_{i}, e_{i}, f_{i}\right)$ in Eqs (32), (33), (34). This solution can be written down in a compact form as

$$
\left[\begin{array}{l}
\boldsymbol{K}_{1} \\
\boldsymbol{K}_{2} \\
\boldsymbol{K}_{3}
\end{array}\right]=\left[\begin{array}{ccc}
1 & 0 & 0 \\
Z_{2}\left(-F_{3}\right) & 1 & 0 \\
Z_{2}\left(F_{2}\right) & Z_{2}\left(-F_{3}\right) & 1
\end{array}\right]\left[\begin{array}{c}
\boldsymbol{I} \\
\boldsymbol{N} \\
\boldsymbol{N}^{2}
\end{array}\right] .
$$

Summarizing, the operators (37) are Haantjes operators that provide the MagriHaantjes chain (29) for any solution $\boldsymbol{N}$ of Eqs. (30), (31) and (35). A simple solution of Eqs. (30) and (31), which leaves invariant both $T S_{1}$ and $\operatorname{Span}\left\{Z_{1}, Z_{2}\right\}$, is

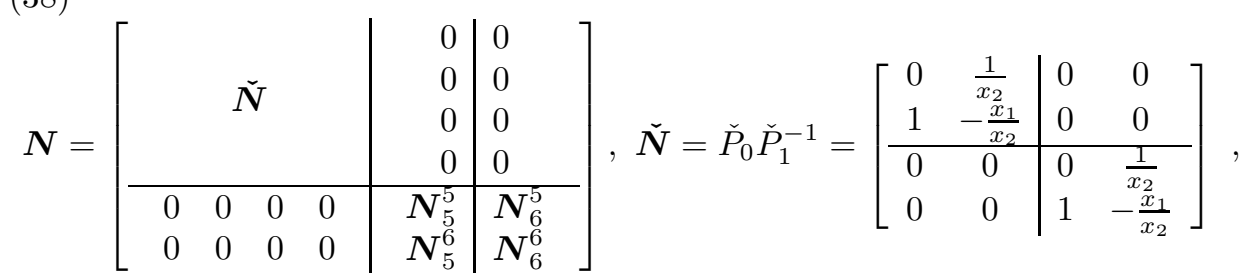

were $\left(\boldsymbol{N}_{5}^{5}, \boldsymbol{N}_{6}^{5}, \boldsymbol{N}_{5}^{6}, \boldsymbol{N}_{6}^{6}\right)$ are arbitrary functions to be determined requiring that the Nijenhuis torsion of $\boldsymbol{N}$ identically vanishes. However, we can postpone the solution of the PDE (35), adding to the system (30)-(35) a further algebraic request, namely that $\boldsymbol{N}$ satisfies the following Magri-Haantjes chain of 1-forms,

(39) $\left(Z_{2}\left(-F_{3}\right) \boldsymbol{I}+\boldsymbol{N}\right)^{T} \mathrm{~d}\left(-F_{3}\right)=\mathrm{d} F_{2},\left(Z_{2}\left(F_{2}\right)+Z_{2}\left(-F_{3}\right) \boldsymbol{N}+\boldsymbol{N}^{2}\right)^{T} \mathrm{~d}\left(-F_{3}\right)=0$. 
Eqs. (39) are independent of Eqs. (32)-(34) as, due to the kernel of the Poisson operator, a Magri-Haantjes chain of Hamiltonian vector fields do not imply that the Magri-Haantjes chain of the gradients of their Hamiltonian functions holds true. Thus, substituting (38) into Eqs. (39), we find the unique solution

$$
\begin{gathered}
\boldsymbol{N}_{5}^{5}=\frac{(c-1) F_{1}+x_{1}}{\Delta}, \quad \boldsymbol{N}_{6}^{5}=\frac{1}{2 c x_{2} \Delta} \\
\boldsymbol{N}_{5}^{6}=-\frac{2 c x_{2}\left((c-1)\left(F_{1}^{2}+F_{1} x_{1}\right)-x_{2}\right)}{\Delta} \\
\boldsymbol{N}_{6}^{6}=-\frac{x_{1}^{3}+(c-1) F_{1} x_{1}^{2}+2 x_{1} x_{2}+(c-1) F_{1} x_{2}}{x_{2} \Delta}
\end{gathered}
$$

where $\Delta=x_{1}^{2}+(c-1) F_{1} x_{1}+x_{2}$. Now, we can verify that it satisfies also Eq. (35), therefore we have got a solution of the problem (27)-(29), which solves also Eqs. (39). It has its minimal polynomial of degree 2

$$
m_{N}(\lambda, \boldsymbol{x})=\lambda^{2}+\frac{x_{1}}{x_{2}} \lambda-\frac{1}{x_{2}}=\lambda^{2}+Z_{2}\left(-F_{3}\right) \lambda+Z_{2}\left(F_{2}\right),
$$

therefore it is a cyclic generator of a Haantjes algebra of rank 2. Moreover, the coefficients of (40) coincides with the elements of the last row in the matrix of (37), consequently $\boldsymbol{K}_{3}=0$.

5.2.6. Restriction to the symplectic leaves of $P_{1}$. From the previous steps it follows

Proposition 17. The deformed Poisson pencil $Q-\lambda P_{1}$ restricts to the symplectic leaves of $P_{1}$. Moreover, $\boldsymbol{N}, \boldsymbol{K}_{1}, \boldsymbol{K}_{2}$, the Hamiltonian functions and the Hamiltonian vector fields $\left(X_{1}, X_{2}\right)$ restrict as well. They endow the symplectic leaves of $P_{1}$ with a $\omega \mathcal{H}$ structure and the Magri-Haantjes chain of exact forms, given by

$$
\check{\boldsymbol{K}}_{1}^{T} \mathrm{~d}\left(-F_{3}\right)_{\mid S_{1}}=\mathrm{d}\left(-F_{3}\right)_{\mid S_{1}}, \quad \check{\boldsymbol{K}}_{2}^{T} \mathrm{~d}\left(-F_{3}\right)_{\mid S_{1}}=\mathrm{d} F_{2_{\mid S_{1}}},
$$

in virtue of (39).

In particular, the relations (37) restricts to the Benenti relations

$$
\check{\boldsymbol{K}}_{1}=\check{\boldsymbol{I}}, \quad \check{\boldsymbol{K}}_{2}=\frac{x_{1}}{x_{2}} \check{\boldsymbol{I}}+\check{\boldsymbol{N}},
$$

as can be immediately seen from the analysis of the minimal polynomial of $\check{N}$ that is still equal to (40). Such relations are satisfied by the so-called $\boldsymbol{L}$-systems [2, 3, 4, proved to be projections of Quasi-Bi-Hamiltonian [20, 21, 28, systems constructed in Riemannian manifolds 29.

5.2.7. Separation of variables. Let us construct in $S_{1}$ a set of Darboux-Haantjes $(\mathrm{DH})$ coordinates for the $\omega \mathcal{H}$ manifold $\left(S_{1}, P_{1}, \check{\boldsymbol{K}}_{1}, \check{\boldsymbol{K}}_{2}\right)$. As the $\omega \mathcal{H}$ structure, in this case, is equivalent to a $\omega N$ structure, as DH coordinates we can take just a set of Darboux-Nijenhuis coordinates [8]. To this aim, we proceed according to the Remark 70, Sect. 8 of [26]. Firstly, we choose as first two coordinates $\left(\lambda_{1}, \lambda_{2}\right)$ just the two (double) eigenvalues of the Haantjes operator $\check{\boldsymbol{K}}_{2}$

$$
\lambda_{1}=\frac{x_{1}-\sqrt{x_{1}^{2}+4 x_{2}}}{2 x_{2}}, \quad \lambda_{2}=\frac{x_{1}+\sqrt{x_{1}^{2}+4 x_{2}}}{2 x_{2}},
$$

as their gradients are eigenforms of $\check{\boldsymbol{K}}_{2}^{T}$

$$
\check{\boldsymbol{K}}_{2}^{T} \mathrm{~d} \lambda_{1}=\lambda_{2} \mathrm{~d} \lambda_{1}, \quad \check{\boldsymbol{K}}_{2}^{T} \mathrm{~d} \lambda_{1}=\lambda_{2} \mathrm{~d} \lambda_{1}
$$


Let us note that $\left(\lambda_{1}, \lambda_{2}\right)$ are also the only eigenvalues of $\boldsymbol{K}_{2}=Z_{2}\left(-F_{3}\right) \boldsymbol{I}+\boldsymbol{N}$, which, therefore, can be used to find half of the separation variables.

Further, we complete them with a pair of conjugate momenta

$$
\mu_{1}=\frac{1}{\lambda_{1}}\left(\lambda_{2} y_{1}+y_{2}\right) \quad \mu_{2}=\frac{1}{\lambda_{2}}\left(\lambda_{1} y_{1}+y_{2}\right)
$$

whose gradients are also eigenforms of $\check{\boldsymbol{K}}_{2}$

$$
\check{\boldsymbol{K}}_{2}^{T} \mathrm{~d} \mu_{1}=\lambda_{2} \mathrm{~d} \mu_{1}, \quad \check{\boldsymbol{K}}_{2}^{T} \mathrm{~d} \mu_{1}=\lambda_{2} \mathrm{~d} \mu_{2} .
$$

The local chart $\left(\lambda_{1}, \lambda_{2}, \mu_{1}, \mu_{2}\right)$ so constructed is a Darboux chart for the Poisson operator $\check{P}_{1}$ and a Haantjes chart for the Haantjes operator $\check{\boldsymbol{K}}_{2}$. In fact, in such chart, they take the form

$$
\check{P}_{1}=i\left[\begin{array}{cc|cc}
0 & 0 & 1 & 0 \\
0 & 0 & 0 & 1 \\
\hline-1 & 0 & 0 & 0 \\
0 & -1 & 0 & 0
\end{array}\right], \quad \check{\boldsymbol{K}}_{2}=\left[\begin{array}{cc|cc}
\lambda_{2} & 0 & 0 & 0 \\
0 & \lambda_{1} & 0 & 0 \\
\hline 0 & 0 & \lambda_{2} & 0 \\
0 & 0 & 0 & \lambda_{1}
\end{array}\right]
$$

(To be more precise, in order to have a Darboux-Haantjes chart one should eliminate the factor $i$ in the form of $\check{P}_{1}$ above, by means of the map $\lambda \mapsto i \lambda, \mu \mapsto \mu$.)

As a consequence of Theorem 59 in [26, the coordinates $\left(\lambda_{1}, \lambda_{2}, \mu_{1}, \mu_{2}\right)$ are separation variables for the functions $F_{2}$ and $F_{3}$. Furthermore, they are also separation variables for the (restriction to $S_{1}$ of the) Hamiltonian function of the Lagrange top

$$
h_{1 \mid S_{1}}=-F_{3 \mid S_{1}}-(c-1) C_{1} F_{2 \mid S_{1}} .
$$

This fact can be proved by means of the Benenti test [1], or simply by observing that, thanks to Eq. (41), it holds true that

$$
\mathrm{d} h_{1 \mid S_{1}}=\left(-\check{\boldsymbol{I}}+(c-1) C_{1} \check{\boldsymbol{K}}_{2}^{T}\right) \mathrm{d} F_{3 \mid S_{1}} .
$$

Therefore the function $h_{1 \mid S_{1}}$ belongs to a Magri-Haantjes chain generated by $F_{3 \mid S_{1}}$. Consequently, according to Theorem 59 in [26], also $h_{1 \mid S_{1}}$ is separable in any $D H$ local chart.

\section{Future Perspectives}

It would be interesting to construct a Poisson-Haantjes algebra for the Poisson pencil $P_{2}-\lambda P_{1}$ of the Lagrange top, that unlike the pencil $P_{0}-\lambda P_{1}$, has two polynomial Casimir functions of the same length 2. Moreover, a Haantjes algebra for the stationary flows [6] of the Boussinesq hierarchy, that also are GZ systems of corank 2 , should be worked out.

\section{ACKNOWLEDGEMENT}

The author is a member of the Gruppo Nazionale di Fisica Matematica (GNFM) of INDAM.

He wishes to thank many useful discussions with his colleagues and friends S. Logar and D. Portelli. Also, many thanks are due to an anonymous referee for the careful reading of the manuscript and for valuable comments and stimulating questions. 


\section{REFERENCES}

[1] S. Benenti, Separability structures on Riemannian manifolds, Lect. Notes Math. 836512 538, Springer, Berlin (1980).

[2] S. Benenti, Inertia tensors and Stackel systems in the Euclidean spaces, Rend. Sem. Mat. Univ. Politec. Torino 50, 315-341 (1992).

[3] S. Benenti, Orthogonal separable dynamical Systems, Math. Publ., Silesian Univ. Opava 1 , 163-184 (1993).

[4] S. Benenti, Special symmetric two-tensors, equivalent dynamical systems, cofactor and bicofactor systems, Acta Appl. Math. 87, 33-91 (2005).

[5] O. I. Bogoyavlenskij, General algebraic identities for the Nijenhuis and Haantjes tensors, Izvestya Mathematics 68, 1129-1141 (2004).

[6] G. Falqui, F. Magri and G. Tondo, Bi-Hamiltonian systems and separation of variables: an example from the Boussinesq hierarchy, Theor. Math. Phys. 122, 176-192 (2000).

[7] G. Falqui, M. Pedroni, On a Poisson reduction for Gelfand-Zakharevich manifolds, Rep. Math. Phys. 50 395-407 (2002).

[8] G. Falqui and M. Pedroni, Separation of variables for bi-Hamiltonian systems, Math. Phys., Anal. and Geom. 6, 139-179 (2003).

[9] A. Frolicher and A. Nijenhuis, Theory of Vector-Valued Differential Forms. Part I, Indag. Mathematicae 18, 338-359 (1956).

[10] I. M. Gelfand, I. Zakharevich, Webs, Lenard schemes, and the local geometry of biHamiltonian Toda and Lax structures, Selecta Math. 6, 131-183 (2000).

[11] J. Haantjes, On $X_{n-1}$-forming sets of eigenvectors, Indag. Mathematicae 17, 158-162 (1955).

[12] Y. Kosmann-Schwarzbach, Beyond recursion operator, Preprint, arXiv :1712.08908v1(2017).

[13] Y. Kosmann-Schwarzbach, F. Magri, Poisson-Nijenhuis structures, Ann. Inst. H. Poincar Phys. Thor. 53, 3581 (1990).

[14] F. Magri, Recursion operators and Frobenius manifolds, SIGMA 8, paper 076, 7 pp. (2012).

[15] F. Magri, Haantjes manifolds, Journal of Physics: Conference Series 482, paper 012028, 10 pp. (2014).

[16] F. Magri, WDVV Equations, Preprint arXiv:1510.07950 (2015).

[17] F. Magri, Haantjes manifolds and Veselov Systems, Theor. Math. Phys. 189, 1486-1499 (2016).

[18] F. Magri, C. Morosi, Characterization of Integrable Systems through the Theory of PoissonNijenhuis Manifolds, Quaderno S 19, Università di Milano (1984).

[19] J. E. Marsden, T. Ratiu, Reduction of Poisson Manifolds, Lett. Math. Phys. 11, 161-169 (1986).

[20] C. Morosi, G. Tondo, Quasi-bi-Hamiltonian systems and separability, J . Phys. A 30, 2799$2806(1997)$.

[21] C. Morosi, G. Tondo, On a class of dynamical systems both quasi-bi-Hamiltonian and biHamiltonian, Phys. Lett. A 247, 59-64 (1998).

[22] C. Morosi, G. Tondo, The quasi-bi-Hamiltonian formulation of the Lagrange top, J. Phys. A 35, 1741-1750 (2002).

[23] C. Morosi, G. Tondo, Separation of Variables in multi-Hamiltonian systems: an application to the Lagrange top, Theor. Math. Phys. 137, 1550-1560, (2003).

[24] A. Nijenhuis, $X_{n-1}$-forming sets of eigenvectors, Indag. Mathematicae 54, 200-212 (1951).

[25] A. Nijenhuis, Jacobi-type identities for bilinear differential concomitants of certain tensor fields I,II, Indag. Math 17, 390-397, 398-403 (1955).

[26] P. Tempesta, G. Tondo, Haantjes Manifolds of Classical Integrable Systems, Preprint arXiv: $1405.5118 \mathrm{v} 2,2016$.

[27] P. Tempesta, G. Tondo Haantjes Algebras and Diagonalization, Preprint, arXiv :1710.04522 (2017)

[28] G. Tondo, C. Morosi, Bi-Hamiltonian manifolds, quasi-bi-Hamiltonian systems and Separation of Variables, Rep. Math. Phys. 44, 255-266 (1999).

[29] G. Tondo, P. Tempesta, Haantjes structures for the Jacobi-Calogero model and the Benenti Systems, SIGMA 12, 023, 18 pp. (2016)

[30] A.V. Tsiganov, On bi-Hamiltonian geometry of the Lagrange top, J. Phys. A: Math. Theor. 41, 315212, 12 pp. (2008). 
Dipartimento di Matematica e Geoscienze, Università degli Studi di Trieste, piaz.le Europa 1, I-34127 Trieste, Italy.

E-mail address: tondo@units.it 